tential) of $\mathrm{Mg}\left(3 s 3 p^{1} P_{1}\right)$, the analogous ${ }^{1} B_{2} \mathrm{MgH}_{2}$ surface is likely to be highly ionic and attractive, and will be the entrance channel for the insertive rotational component, but end-on attack could also proceed with some efficiency. Experimental results ${ }^{13,14}$ and theoretical calculation $\mathrm{s}^{13-15}$ for the $\mathrm{Li}\left({ }^{2} P\right)-\mathrm{H}_{2}$ and $\mathrm{Na}\left({ }^{2} P\right)-\mathrm{H}_{2}$ sy stems have also indicated the importance of similarly attractive ${ }^{2} B_{2}$ surfaces with substantial ionic character.

The rotational distributions of $\operatorname{MgH}(v=0)$ resulting from the reaction of $\operatorname{Mg}\left({ }^{1} P_{1}\right)$ with a whole range of hy drocarbons ${ }^{16}$ are virtually identical and are similar to the abstractive branching component of the $\mathrm{Mg}\left({ }^{1} P_{1}\right)-\mathrm{H}_{2}$ reaction. Steric hindrance and the absence of strong charge-transfer interactions appear to favor a direct end-on attack of alkyl $\mathrm{C}-\mathrm{H}$ bonds by $\operatorname{Mg}\left({ }^{1} P_{1}\right)$.

a) Research supported by the National Science Foundation and, in part, by the Department of Energy through the Utah Consortium for Energy Research and Education.

${ }^{1}$ I. W. M. Smith, Kinetics and Dynamics of Elementary Gas Reactions (Butterworths, London, 1980).

${ }^{2}$ M. R. Levy, Prog. React. Kinet. 10, 1 (1979).

${ }^{3} \mathrm{~W}$. H. Breckenridge and H. Umemoto, "Collisional quenching of electronically excited atoms," Adv. Chem. Phys. (in press).

AA. Gupta, D. S. Perry, and R. N. Zare, J. Chem. Phys. 72,
$6237,6250(1980)$, and references therein.

${ }^{5}$ F. E. Bartoszek, B. A. Blackwell, J. C. Polanyi, and J. J. Sloan, J. Chem. Phys. 74, 3400 (1981), and references therein.

${ }^{6}$ A. C. Luntz, R. Schinke, W. A. Lester, Jr., and Hs. H. Günthard, J. Chem. Phys. 70, 5908 (1979); A. C. Luntz, ibid. 73, $1143(1980)$; A. C. Luntz and P. Andresen, ibid. $72,5842,5851$ (1980).

${ }^{7}$ G. K. Smith and J. E. Butler, J. Chem. Phys, 73, 2243 $(1980)$, and references therein.

${ }^{8}$ J. C. Polanyl, J. L. Schreiber, and W. J. Skrinc, Discuss. Faraday Soc. 67, 66 (1979); J. C. Polanyi and W. J. Skrlas, Chem. Phys. 23, 167 (1977); D. Brandt and J. C. Polanyi, ibid. 35, 23 (1978).

${ }^{9}$ W. H. Breckenridge and H. Umemoto, J. Chem. Phys. 75, 698 (1981).

${ }^{10}$ W. H. Breckenridge, O. Kim Malmin, W. L. Nikolai, and D. Oba, Chem. Phys. Lett. 59, 38 (1978).

${ }^{11}$ W. J. Balfour and H. M. Cartwright, Astron. Astrophys. Suppl. Ser. 26, 389 (1976).

${ }^{12}$ N. Adams, W. H. Breckenridge, and Jack Simons, Chem. Phys. 56, 327 (1981):

${ }^{13}$ P. Botschwina, W. Meyer, I. Hertel, and W. Relland (submitted).

${ }^{14}$ I. V. Hertel, "Progress in electronic to vibrational energy transfer," Adv. Chem. Phys. (in press).

${ }^{15} \mathrm{~K}$. Mizutami, Y. Kuribara, K. Hayashi, and S. Matsumoto, Bull. Chem. Soc. Jpn. 62, 2184 (1979).

${ }^{16} \mathrm{~W}$. H. Breckenridge and $\mathrm{H}$. Umemoto (to be submitted).

${ }^{17}$ K. -H. Gericke, F. J. Comes, and R. D. Levine, J. Chem. Phys. 74, 6106 (1981).

\title{
Coverage-dependent surface enhanced Raman spectra of halide ions on colloidal silver
}

\author{
Robin L. Garrell, Keith D. Shaw, and Samuel Krimm \\ Department of Physics and Macromolecular Research Center, The University of Michigan, Ann Arbor, \\ Michigan 48109 \\ (Received 28 July 1981; accepted 13 August 1981)
}

Surface enhanced Raman spectroscopy (SERS) provides a sensitive method for observing vibrational excitations of molecules that interact with metal surfaces. Theoretical studies of this effect suggest that the observed frequencies and scattered intensities are a function of the extent, density, and homogeneity of surface coverage. ${ }^{1-4}$ Some experimental studies support these predictions, such as frequency shifts of halide ion bands observed as a function of applied voltage in electrochemical cells ${ }^{5}$ and coverage-dependent intensity variations seen for pyridine adsorbed on $\mathrm{Ag}$ surfaces prepared in ultrahigh vacuum. ${ }^{3}$ In the case of halide ions, numerous observations $\mathrm{s}^{5-9}$ of a band near $240 \mathrm{~cm}^{-1}$ have been interpreted by Nichols and Hexter ${ }^{4}$ as arising from a $\mathrm{Ag}-\mathrm{Cl}^{-}$stretching vibration, and they have predicted that this frequency should vary continuously as the surface coverage changes from complete to single atom. Similar predictions were made for the other halide ions."
We have obtained SERS spectra of $\mathrm{Cl}^{-}, \mathrm{Br}^{-}$, and $\mathrm{I}^{-}$as a function of coverage on $\mathrm{Ag}$ sols, and find agreement with some of the predictions of Nichols and Hexter. However, we also find that the observed bands show a definite fine structure, which we believe can be attributed in part to the discrete nature of surface coverage structures.

Our samples were prepared by adding 1-50 $\mu 1$ amounts of $0.1-15.0 \mathrm{mM}$ haloacids to $0.5 \mathrm{ml}$ portions of $\mathrm{Ag}$ sols prepared following the procedure of Creighton et al. ${ }^{10}$ Electron micrographs of this preparation show faceted Ag particles about $300 \AA$ in diameter. Raman spectra were obtained at a bandwidth of $\sim 1 \mathrm{~cm}^{-1}$ on a spectrometer ${ }^{11}$ to which data acquisition capabilities (Cromemco Z-2 microcomputer system) had been added. Further experimental details will be given in a subsequent publication. ${ }^{12}$ 


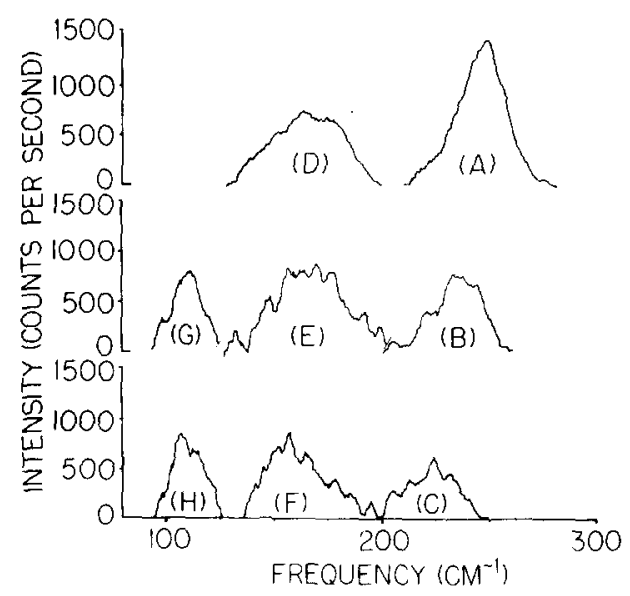

FIG. 1. Raman spectra of halides in aqueous silver sols: (A) maximum coverage $\mathrm{Cl}^{-}$; (B) partial coverage $\mathrm{Cl}^{-}$obtained in the presence of trace $\mathrm{Br}^{-}$; (C, F) less than monolayer coverage of $\mathrm{Br}^{-}$with excess $\mathrm{Cl}^{-}$present; (D) maximum coverage $\mathrm{Br}^{-}$; (E) partial $\mathrm{Br}^{-}$coverage in mixed $\mathrm{Br}^{-}, \mathrm{Cl}^{-}$experiment; $(\mathrm{G}, \mathrm{H})$ partial coverage $\mathrm{I}^{-}$(unranked). All spectra taken at $100-200 \mathrm{~mW}$, 4 scans, $1 \mathrm{~cm}^{-1}$ bandwidth, 17 point smoothing function and background subtraction applied.

In Fig. 1 we show SERS spectra of $\mathrm{Cl}^{-}, \mathrm{Br}^{-}$, and $\mathrm{I}^{-}$ (we did not succeed in obtaining spectra of $\mathrm{F}^{-}$, probably because of the high solubility of $\mathrm{AgF}$ ). Our maximum coverage spectra were obtained by adding the equivalent of at least ten times the amount of haloacid required for complete monolayer coverage (as estimated from the total Ag surface area in the sol). Partial coverage was achieved either by adding an appropriate fraction of this amount or by replacement of one ion by another. We find, for example, that if $\mathrm{HBr}$ is added to a maximum coverage $\mathrm{Cl}^{-}$sol, the $\mathrm{Cl}^{-}$band diminishes in time with a concomitant intensity increase in the $\mathrm{Br}^{-}$band. ${ }^{11}$ While absolute partial coverage percentages were not obtainable, such experiments permitted us to rank spectra in order of coverage.

The maximum coverage spectrum of $\mathrm{Cl}^{-}$sols [Fig. $1(A)]$ shows a single band whose peak is at $245 \mathrm{~cm}^{-1}$, in reasonable agreement with the value of $241 \mathrm{~cm}^{-1}$ calculated for full coverage of $\mathrm{Cl}^{-}$on a $\mathrm{Ag}(100)$ face. ${ }^{4}$ This band, however, has shoulders on the low and high frequency sides, the latter being found near $252 \mathrm{~cm}^{-1}$. At less than maximum coverage the lower frequency components stand out clearly [Figs. $1(B)$ and $1(C)$, the maximum intensity shifting to lower frequencies with decreasing coverage. The positions of some of these components are essentially constant, while those of others shift slightly with coverage: 205(B), 205(C); 215(A), 213(C); 220(B); 224(B), 224(C); 231(A), 232(B), 231(C); 238(A), 236(B), 234(C); 245(A), 242(B), 240(C); 252(A), 249(B).

The maximum coverage spectrum of $\mathrm{Br}^{-}$[Fig. 1(D)] similarly shows a single band with shoulders. Its peak frequency near $170 \mathrm{~cm}^{-1}$ is higher than the calculated (100) full coverage frequency ${ }^{4}$ of $150 \mathrm{~cm}^{-1}$. The downward frequency shift in the intensity maximum with decreasing coverage and the appearance of fine structure at partial coverage are clearly evident [Figs. 1(E) and
$1(F)$. The number of components is larger than for $\mathrm{Cl}^{-}$, and the detailed matching is less clear: (D) 139,153 , $164,175,189$; (E) 132, 141, 146, 157, 162, 169, 176, $181,189,198$; (F) $142,146,153,158,166,170,178$, $186,195$.

The spectra of $\mathrm{I}^{-}$sols [Figs. 1(G) and 1(H)] show analogous but less pronounced effects. The highest frequency maximum occurs at $113 \mathrm{~cm}^{-1}$, the same as the calculated (100) full coverage frequency. ${ }^{4}$ Fine structure components are seen at lower and higher frequencies: (G) $100,107,113,121$; (H) 99, 108, 115, 117, 121,123 .

The theory of Nichols and Hexter ${ }^{4}$ is based on coupling within a monolayer of vibrating dipoles consisting of real-plus-image charges, and predicts a continuously decreasing frequency with decreasing surface coverage, from the full coverage value to the static field frequency of a single ion $\left(159,100\right.$, and $82 \mathrm{~cm}^{-1}$ for $\mathrm{Cl}^{-}, \mathrm{Br}^{-}$, and $\mathrm{I}^{-}$, respectively). Our results indeed show a downward frequency shift in the overall band center as coverage decreases, although we are not able to quantitate this dependence. What is even more interesting, however, is that our bands show a fine structure: each observed band appears to be a sum of components whose frequencies are (or nearly are) constant, but whose relative intensities vary, with surface coverage. This result is not unexpected.

In the above theory ${ }^{4}$ lattice dipole sums at intermediate coverage are obtained by multiplying the full coverage lattice sum by the coverage fraction. In reality the lattice sums vary discretely and are affected primarily by the near-neighbor dipole arrangements: changes in nearest neighbor site occupancy cause large discrete changes in the frequency of a given dipole, while smaller changes about these values result from occupancy variations at more distant sites. Of course, the actual frequencies depend on the value of the full coverage frequency, which is a function of the packing of ions on the particular Ag plane [e.g., (100) or (111)].

Using this model, we have been able ${ }^{13}$ to calculate the approximate frequency distribution to be expected as a function of coverage fraction. There is good enough general agreement between peaks in these distributions and those in our spectra to suggest that the observed fine structure arises from specific local arrangements of halide ions on the $\mathrm{Ag}$ surfaces.

We are indebted to G. W. Ford and W. H. Weber for helpful discussions. This research was supported by National Science Foundation grants DMR 78-00753 and PCM 79-21652, and by the Macromolecular Research Center (R. L. G.).

${ }^{1}$ G. D. Mahan and A. A. Lucas, J. Chem. Phys. 68, 1344 (1978).

${ }^{2}$ G. L. Eesley and J. R. Smith, Solid State Commun. 31, 815 (1979).

${ }^{3}$ G. L. Eesley, Physics Lett. A 81, 193 (1981).

${ }^{4}$ H. Nichols and R. M. Hexter, J. Chem. Phys. 74, 2059 (1981).

${ }^{5} \mathrm{H}$. Wetzel, H. Gerischer, and B. Pettinger, Chem. Phys. Lett. 78, 392 (1981). 
${ }^{6}$ J. A. Creighton, M. G. Albrecht, R. E. Hester, and J. A. D. Matthew, Chem. Phys. Lett. 55, 55 (1978).

${ }^{7}$ A. Otto, Surf. Scl. 75, 392 (1978).

${ }^{8}$ J. R. Lombardi, E. A. Shields Knight, and R. Birke, Chem. Phys. Lett. 79, 214 (1981).

${ }^{9}$ M. E. Lippitsch, Chem. Phys. Lett. 74, 125 (1980).
${ }^{10}$ J. A. Creighton, G. C. Blatehford, and M. G. Albrecht, J. Chem. Soc. Faraday Trans. 2 75, 790 (1979).

${ }^{11}$ S. L. Hsu, W. H. Moore, and S. Krimm, J. Appl. Phys. 46, 4185 (1975).

${ }^{12}$ R. G. Garrell, K. D. Shaw, and S. Krimm (to be published).

${ }^{13}$ K. D. Shaw, R. L. Garrell, and S. Krimm (to be published).

\title{
NOTES
}

\section{Spin-orbit coupling and $\Lambda$ doubling in $\mathrm{NaAr}$}

\author{
David L. Cooper a) \\ Physical Chemistry Labonatory, South Parks Road, Oxford, England OX1 $3 Q Z$ \\ (Received 12 September 1980; accepted 12 November 1980)
}

The van der Waals molecule NaAr has been extensively studied ${ }^{1-7}$ and a considerable amount of spectroscopic information is available. Smalley et al. ${ }^{2}$ have observed $\Lambda$ doubling in the $A^{2} \Pi$ state of this molecule and have attempted, using a rather crude model, to use calculated potentials to account for the observed splittings. This was rather unsuccessful. In this work, we shall reproduce the magnitude and variation with vibrational quantum number of the $\Lambda$-doublet splittings using a more quantitative model. We shall also calculate spin-orbit coupling constants - these are known experimentally but with rather large error limits. Close to its equilibrium geometry, $\operatorname{NaAr}\left(A^{2} \Pi_{r}\right)$ behaves as a good Hund's case (a) molecule and good $a b$ initio values might be expected. However, the system is more complex (in terms of the number of electrons) than systems previously studied. NaAr thus presents an interesting challenge.

The fluorescence excitation spectrum of the $X^{2} \Sigma^{+}-$ $A^{2} \Pi$ optical transition has been measured ${ }^{1,2}$ in a super sonic expansion of sodium in a helium carrier gas containing a few percent argon. The $X^{2} \Sigma^{+}$ground state $\mathrm{Na}\left({ }^{2} S_{1 / 2}\right)+\operatorname{Ar}\left({ }^{1} S_{0}\right)$ is only weakly attractive and only the $v^{\prime \prime}=0$ to $v^{\prime \prime}=4$ levels are known. The $A^{2} \Pi$ state is much more strongly bound and transitions to vibrational levels $v^{\prime \prime}=7$ to $v^{\prime \prime}=11$ have been observed. A mainly repulsive $B^{2} \Sigma^{+}$state has been studied indirectly by the $\Lambda$ doubling in the $A^{2} \Pi_{1 / 2}$ state. These states both dissociate to $\mathrm{Na}\left({ }^{2} P_{1 / 2}\right)+\operatorname{Ar}\left({ }^{1} S_{0}\right)$ and are close in energy. (The $A^{2} \mathrm{II}-B^{2} \Sigma^{+}$interaction should dominate the contributions due to other ${ }^{2} \Sigma$ states.)

In the conventional theory of $\Lambda$-doubling in ${ }^{2} \Pi$ states, the splitting in the $v$ th vibrational level may be expressed to second order in terms of two parameters $\left(\frac{1}{2} p_{v}+q_{v}\right)$ and $q_{v}$, where $\mathrm{e}^{8,9}$

$p_{v}=4 \sum_{n^{\prime} v^{\prime}} \frac{\left.(-1)^{k / 2} \Pi v\left|H_{\mathrm{SO}}\right| n^{\prime} v^{\prime}\right\rangle\left\langle n^{\prime} v^{\prime}\left|B\left(L^{+} S^{-}+L^{-} S^{+}\right)\right|^{2} \Pi v\right\rangle}{E_{\mathrm{\Pi} v}-E_{n^{\prime} v^{\prime}}}$,

$q_{v}=2 \sum_{n^{\prime} v^{\prime}} \frac{\left.(-1)^{k}\left|{ }^{2} \Pi v\right| B\left(L^{+} S^{-}+L^{-} S^{+}\right)\left|n^{\prime} v^{\prime}\right\rangle\right|^{2}}{E_{\Pi v}-E_{n^{\prime} v^{\prime}}}$.
The summations are over all vibrational levels of all ${ }^{2} \Sigma$ states $-k$ is even for $\Sigma^{+}$states and odd for $\Sigma^{-}$states. The denominator in both expressions is the energy separation between the interacting vibronic levels; $B$ is an operator proportional to $1 / \mu R^{2}$, where $\mu$ is the reduced mass and $R$ is the internuclear distance; $L^{+}$and $S^{+}$are the raising operators for orbital and spin angular momenta, respectively; $H_{\text {so }}$ is the spin-orbit coupling operator.

A method due to Hutson et al. ${ }^{10}$ has been previously used $^{11}$ for the direct evaluation of the vibrational summations in the expressions for $p$ and $q$. A quantity $\left|n^{\prime} \omega^{\prime}\right\rangle$ may be obtained such that the expressions reduce to

$$
\begin{aligned}
& p_{v}=4 \sum_{n^{\prime}}(-1)^{k}\left\langle{ }^{2} \Pi v\left|H_{\mathrm{SO}}\right| n^{\prime} \omega^{\prime}\right\rangle, \\
& q_{v}=2 \sum_{n^{\prime}}(-1)^{k}\left\langle 2 \Pi v\left|B\left(L^{+} S^{-}+L^{-} S^{+}\right)\right| n^{\prime} \omega^{\prime}\right\rangle,
\end{aligned}
$$

TABLE I. Spin-orbit coupling matrix elements for the $A^{2}$ II state of NaAr.

\begin{tabular}{cccc}
\hline \hline $\begin{array}{c}\text { Geometry } \\
\text { (in bohr) }\end{array}$ & $\begin{array}{c}\text { One electron } \\
\text { contribution } \\
\text { (in } \text { cm }^{-1} \text { ) }\end{array}$ & $\begin{array}{c}\text { Two electron } \\
\text { contribution } \\
\text { (in } \text { cm }^{-1} \text { ) }\end{array}$ & $\begin{array}{c}\text { Total } \\
\text { (in } \text { cm }^{-1} \text { ) }\end{array}$ \\
\hline 3.0 & 24.30 & -5.18 & 19.12 \\
3.5 & 23.91 & -5.21 & 18.70 \\
4.0 & 22.65 & -5.03 & 17.62 \\
4.5 & 20.89 & -4.74 & 16.15 \\
5.0 & 19.03 & -4.42 & 14.61 \\
5.5 & 17.31 & -4.12 & 13.19 \\
5.7 & 16.69 & -4.01 & 12.67 \\
6.0 & 15.83 & -3.86 & 11.97 \\
6.3 & 15.08 & -3.73 & 11.35 \\
7.0 & 13.70 & -3.48 & 10.21 \\
8.0 & 12.47 & -3.26 & 9.21 \\
9.0 & 11.85 & -3.15 & 8.69 \\
10.0 & 11.55 & -3.10 & 8.43 \\
11.0 & 11.41 & -3.07 & 8.34 \\
\hline \hline
\end{tabular}

